$7-2-2012$

\title{
Geometric Constructions for Image Formation by A Converging Lens
}

Ulrich Zürcher

Cleveland State University, u.zurcher@csuohio.edu

Follow this and additional works at: https://engagedscholarship.csuohio.edu/sciphysics_facpub

Part of the Physics Commons

How does access to this work benefit you? Let us know!

Publisher's Statement

This is an author-created, un-copyedited version of an article accepted for publication in European Journal of Physics. The publisher is not responsible for any errors or omissions in this version of the manuscript or any version derived from it. The Version of Record is available online at http://dx.doi.org/10.1088/0143-0807/33/5/1125

\section{Repository Citation}

Zürcher, Ulrich, "Geometric Constructions for Image Formation by A Converging Lens" (2012). Physics Faculty Publications. 273.

https://engagedscholarship.csuohio.edu/sciphysics_facpub/273

This Article is brought to you for free and open access by the Physics Department at EngagedScholarship@CSU. It has been accepted for inclusion in Physics Faculty Publications by an authorized administrator of EngagedScholarship@CSU. For more information, please contact library.es@csuohio.edu. 


\title{
Geometric constructions for image formation by a converging lens
}

\author{
Ulrich Zürcher
}

\begin{abstract}
Light rays emerge from an object in all directions. In introductory texts, three 'special' rays are selected to draw the image produced by lenses and mirrors. This presentation may suggest to students that these three rays are necessary for the formation of an image. We discuss that the three rays attain their 'special status' from the geometric solution of the equation of a hyperbola $x^{-1}+y^{-1}=c^{-1}$ (mirror/lens equation). The material is suitable for use in introductory courses for science majors.
\end{abstract}

\section{Introduction}

Light rays follow piecewise straight lines governed by the laws of reflection or refraction (Snell's law). Rays emerge from an object (light source) in all directions. Following earlier studies by Euclid, Al-Kindī proposed that 'everything in the world ... emits rays in every direction, which fill the whole world' [1].

In introductory texts, three 'special' rays are selected among the myriad of possible ones, which are then used to find the image formed by a mirror or lens [2, 3]. However, little is said about what makes these three rays so 'special;' this vagueness may lead students to believe that these rays are necessary to form an image. Such a misconception may contribute to students' difficulty in answering conceptual questions, such as explaining image formation in the case where half of the lens is covered [4].

In the paraxial approximation of geometric optics, the object and image distances $d_{o}$ and $d_{i}$ are related to each other by the mirror or lens equation,

$$
\frac{1}{d_{o}}+\frac{1}{d_{i}}=\frac{1}{f}
$$

where $f$ is the focal length. The magnification of the image is determined by

$$
m=\frac{h_{i}}{h_{o}}=-\frac{d_{i}}{d_{o}}
$$

where $h_{o}$ and $h_{i}$ are the object and image heights, respectively. Equation (1) is the equation of a hyperbola $x^{-1} \quad y^{-1} \quad c^{-1}$ that can be solved with geometric constructions using a 
ruler and compass $[5,6]$. In fact, the three rays attain their 'special status' from the Euclidean construction of the solution of the mirror/lens equation.

In our (algebra-based) introductory course, we emphasize the connection between 'special rays' and the geometric solution of the mirror/lens equation by solving non-trivial problems of image formation. This type of problem solving has the additional benefit of illustrating to students how one 'can tell a lot about the lens and the images from the given value of $m$ ' [2]? We use the method for image formation by a convex lens; similar solutions for a diverging lens and a convex/concave mirror are possible. We solve the problems step-by-step and compare the algebraic manipulations with the corresponding geometrical constructions.

We use the notation and convention in [3]. A lens has focal points $F_{1}$ and $F_{2}$ in front of and behind the lens, respectively. The focal length of a converging lens is positive $f>0$. The object is in front of the lens so that we always have $d_{o}>0$. If the image is behind (in front of) the lens, then $d_{i}>0\left(d_{i}<0\right)$. We can always take the object height to be positive $h_{o}>0$. The image height is positive $h_{i}>0$ and negative $h_{i}<0$ for upright (virtual) and inverted (real) images, respectively. We define the distance between the object and the image as a non-negative number $\Delta=\left|d_{o}+d_{i}\right| \geqslant 0$, independent of the location of the image with respect to the object.

We follow a standard convention for the three 'special' rays [3].

- Ray 1: incident ray parallel to the principal axis: refracted ray through the focal point $F_{2}$.

- Ray 2: incident ray through the focal point $F_{1}$ : refracted ray parallel to the principal axis.

- Ray 3: Straight line through the tip of the object and centre of the lens.

We also need to draw circles with radius $\sqrt{a b}$, where $a$ and $b$ are lengths depending on the situation. The corresponding geometric construction is discussed in [5, 6]. However, this step can be skipped without any loss of physical insight. We use this simplification, and draw a circle with a radius equal to the numerical value of $\sqrt{a b}$.

The lens equation is a relation between three lengths $d_{o}, d_{i}$ and $f$ so that two lengths determine the remaining one. These problems are trivial and are not considered here. We discuss the problem when the distance between the object and distance $\Delta$ and the magnification $m$ are given. In the usual paraxial approximation of geometrical optics, the height of the object $h_{o}$ is arbitrary, and the magnification determines the image height $h_{i}$. Thus, the distance $\Delta$ and the height $h_{i}$ are the two lengths characterizing the problem, and we seek the object and image distance, $d_{o}$ and $d_{i}$, and the focal length $f$. We then discuss the problem when magnification $m$ and the focal length are given, and find the object and image distances $d_{o}$ and $d_{i}$. These two problems can be solved using a ruler only. Finally, the case when the distance $\Delta$ and the focal length $f$ are given involves the solution of a quadratic equation. The corresponding geometric solution involves the use of a ruler and a compass. While the geometric constructions presented here are not new, they are generally not discussed in current texts.

\section{Solution with a ruler only}

Problems in which the image has a known magnification $m$ can be solved with a ruler only. The order of drawing the three rays differ in these two problems. For each step, we write down the corresponding step in the algebraic solution.

\section{Given $m$ and $\Delta$}

Problem. Given the magnification $m$ and the distance $\Delta$ between object and image, find the focal length $f$ and the location of the lens relative to the object (or image). Numerical values: $m=3$ and $\Delta=4.0 \mathrm{~cm}$. 

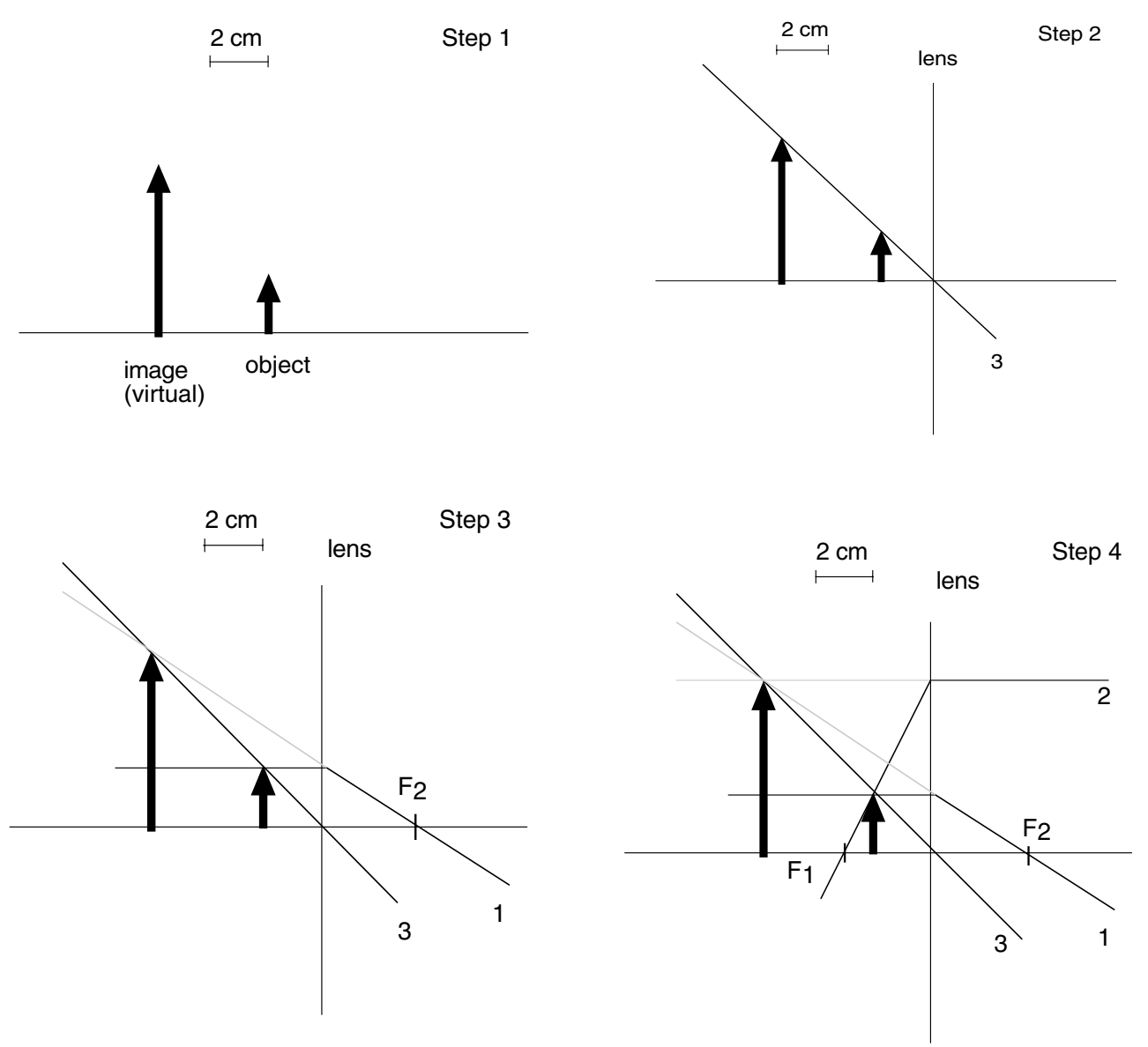

Figure 1. The geometric construction for finding the object and image distances $d_{o}$ and $d_{i}$ and the focal length $f$ of a converging lens, if the magnification of the image and the distance between object and distance are known. The construction applies to the case of a positive magnification, i.e. a virtual image.

Solution. We choose $h_{o}=2.0 \mathrm{~cm}$ so that $h_{i}=6.0 \mathrm{~cm}$.

Step 1. Since $m=-d_{i} / d_{0}=3$, it follows that $d_{i}=-3 d_{o}$ : the image is virtual and is farther away from the lens than the object-figure 1 (step 1).

Step 2. We draw ray 3 that connects the tips of the object and image. The intercept with the principal axis gives the location of the lens-figure 1 (step 2).

We have $h_{o} / d_{o}=\left(h_{i}-h_{o}\right) / \Delta$, so that the object distance follows

$$
d_{o}=\frac{h_{o}}{h_{i}-h_{o}} \Delta=\frac{\Delta}{m-1} .
$$

We find the numerical value $d_{o}=4.0 \mathrm{~cm} /(3-1)=2.0 \mathrm{~cm}$, and the image distance $d_{i}=-d_{o}-\Delta$,

$$
d_{i}=-\frac{h_{i}}{h_{i}-h_{o}} \Delta=-\frac{\Delta}{1-m^{-1}}
$$

so that $d_{i}=-4.0 \mathrm{~cm} /\left(1-3^{-1}\right)=-(3 / 2) \cdot 4.0 \mathrm{~cm}=-6.0 \mathrm{~cm}$. 
Step 3. We draw the incident ray 1 from the tip of the object to the known lens and find the intercept with the lens. We draw the line from this intercept to the tip of the image; its continuation is the refracted ray 1 . The intercept of the refracted ray 1 with the principal axis gives the focal point $F_{2}$-figure 1 (step 3 ).

We find $\left(h_{i}-h_{o}\right) /\left(-d_{i}\right)=h_{o} / f$ so that

$$
f=-\frac{h_{o}}{h_{i}-h_{o}} d_{i}=\frac{m}{(m-1)^{2}} \Delta .
$$

That is, $f=\left(3 / 2^{2}\right) \cdot 4.0 \mathrm{~cm}=3.0 \mathrm{~cm}>0$.

Step 4 . We draw the refracted ray 2 to the known lens, and then find the intercept with the lens. We draw the line from this intercept to the object; this is the incident ray 2 . The intercept of the continuation of the incident ray 2 with the principal axis gives the focal point $F_{1}$ - figure 1 (step 4).

We note that the focal points $F_{1}$ and $F_{2}$ are symmetrical with respect to the lens.

Discussion: When the magnification approaches 1 from above, $m \rightarrow 1^{+}$, the slope of ray 3 decreases and its intercept with the principal axis moves towards the right so that the lens moves further away from the object and image, and $d_{o}$ and $d_{i}$ increase as a result. We see from ray 1 that the focal length $f$ also increases.

\section{Given $m$ and $f$}

Problem. Given the magnification $m$ and the focal length $f$, find the location of the object $d_{o}$ and the location of the image $d_{i}$. Numerical values: $m=-3 / 2$ and $f=4.0 \mathrm{~cm}$.

Solution. We choose $h_{o}=4.0 \mathrm{~cm}$ so that $h_{i}=-6.0 \mathrm{~cm}$.

Step 1. The image is real and behind the lens. We draw the lines parallel to the principal axis at the heights $h_{o}$ and $h_{i}$-figure 2 (step 1).

Step 2. The incident ray 1 lies on the line at the height $h_{o}$. We find the intercept with the lens. The refracted ray 1 is then drawn from this intercept through the focal point $F_{2}$. The intercept of the refracted ray 1 with the line at height $h_{i}$ gives the location of the image-figure 2 (step 2). We have $h_{o} / f=-h_{i} /\left(d_{i}-f\right)$, or

$$
d_{i}=(1-m) f \text {, }
$$

so that $d_{i}=(1-(-3 / 2)) \cdot 4.0 \mathrm{~cm}=(5 / 2) \cdot 4.0 \mathrm{~cm}=10.0 \mathrm{~cm}$. Note that $m<0$ so that $d_{i}>0$, i.e. the (real) image is behind the lens.

Step 3. The refracted ray 2 lies on the line at the height $h_{i}$. We find the intercept with the lens. The incident ray 2 is then drawn from this intercept through the focal point $F_{1}$. The intercept of the incident ray 2 with the line at height $h_{o}$ gives the location of the object-figure 2 (step 3). We find $-h_{i} / f=h_{o}\left(d_{o}-f\right)$, or

$$
d_{o}=\left(1-m^{-1}\right) f
$$

so that $d_{o}=\left(1-(-3 / 2)^{-1}\right) \cdot 4.0 \mathrm{~cm}=(5 / 3) \cdot 4.0 \mathrm{~cm}=6.7 \mathrm{~cm}$.

Step 4 . We draw ray 3 to confirm the location of the object and the image-figure 2 (step 4).

Discussion: When the magnification approaches 1 from above $|m| \rightarrow 1^{+}$, the intercept of ray 1 with the horizontal line $h_{i}$ moves towards the lens so that $d_{i}$ decreases. The slope of the incoming ray 2 decreases; the object moves away from the lens so that $d_{o}$ increases. We keep $h_{o}$ fixed and decrease $h_{i}$. Then, the slope of the refracted ray 1 is constant, whereas the slope of incoming ray 2 decreases. We see that the increase in $d_{o}$ is smaller than decrease in $d_{i}$. We conclude that the distance $\Delta$ between the object and the image decreases as $|m| \rightarrow 1^{+}$. 

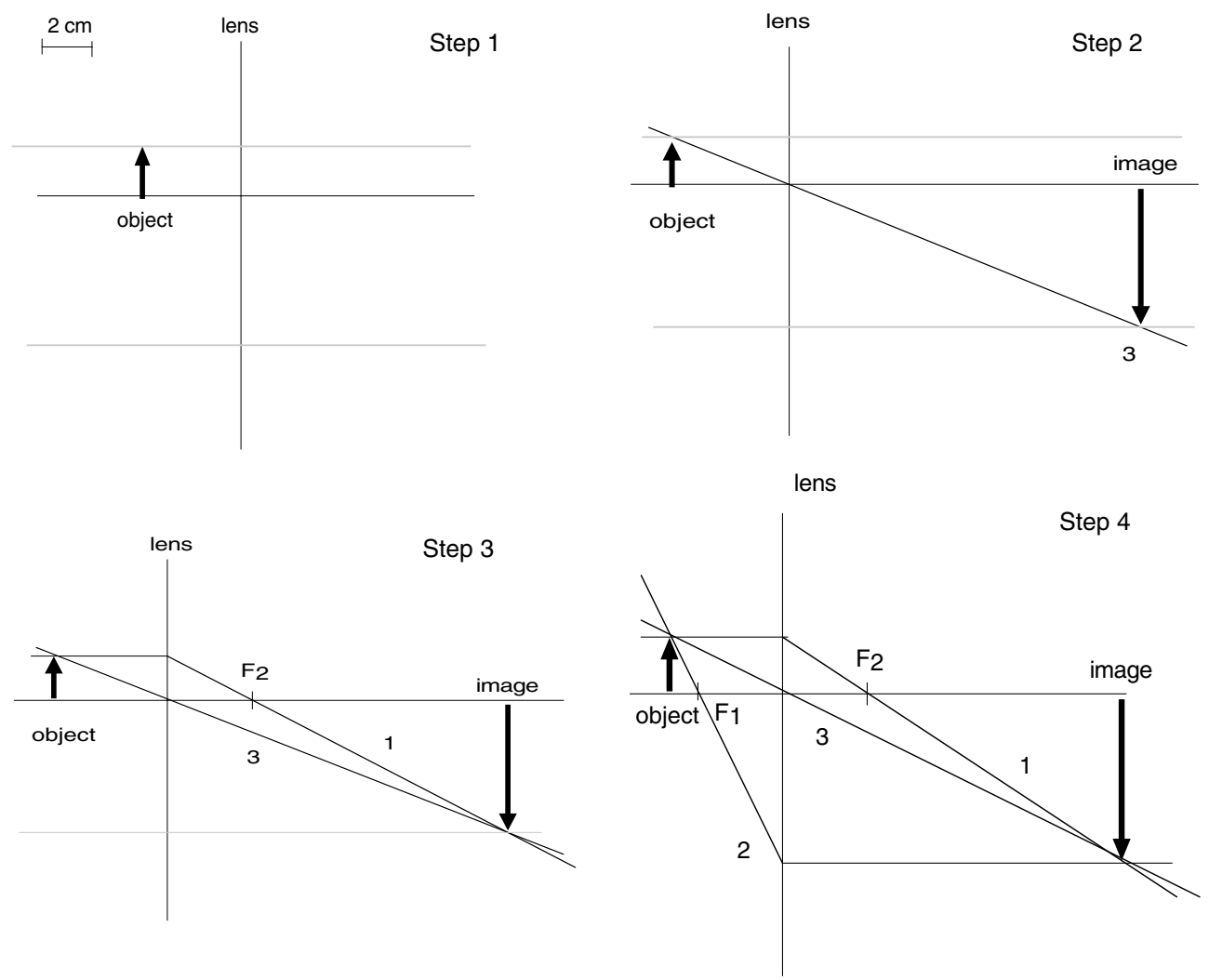

Figure 2. The geometric construction for finding the object and image distances $d_{o}$ and $d_{i}$, if the magnification of the image and the focal length $f$ of a converging lens are known. The construction applies to the case of a negative magnification, i.e. a real image.

\section{Solution with both a ruler and a compass}

A geometric construction with only a ruler requires that rays $1-3$ can be drawn. This is not the case when the distance $\Delta$ between object and image and the focal length $f$ are given. In this case, the construction also requires the use of a compass. When this problem is solved algebraically, the solution involves finding the roots of a quadratic equation. We distinguish between the real and virtual images.

\section{Real image}

We use $\delta$ for the location of the lens relative to the midpoint between the object and the image. We assume without loss of generality that the object distance is greater than the image distance, and write $d_{o}=\Delta / 2+\delta$ and $d_{i}=\Delta / 2-\delta$. The lens equation is then written:

$$
\frac{1}{\Delta / 2+\delta}+\frac{1}{\Delta / 2-\delta}=\frac{1}{f}
$$


The case $\delta=0$ corresponds to the case when the object and image distances are equal, $d_{o}=d_{i}=\Delta / 2$. This gives $2 \cdot(\Delta / 2)^{-1}=4 / \Delta=1 / f$ or for the maximum focal length for a given distance $\Delta$ between the object and the image:

$$
f<f_{\max }=\frac{\Delta}{4} \text {. }
$$

For $f<f_{\max }$, the location of the lens moves away from the object and towards the image.

Problem. Find the location of a lens with focal length $f$ if the distance $\Delta$ between the object and the image is given. Numerical example: $\Delta=16.0 \mathrm{~cm}$ and $f=2.25 \mathrm{~cm}$ so that $\sqrt{f \Delta}=6.0 \mathrm{~cm}$.

Solution. Since the image distance is positive $d_{i}>0$, the distance between the object and image follows $\Delta=d_{o}+d_{i}$. We write $d_{i}=\Delta-d_{o}$, and find $1 / d_{o}+1 /\left(\Delta-d_{o}\right)=1 / f$. It follows $f \Delta=d_{o}\left(\Delta-d_{o}\right)$, or by completing the square $\left(d_{o}-\Delta / 2\right)^{2}=(\Delta / 2)^{2}-f \Delta$,

$$
\delta^{2}=\left(\frac{\Delta}{2}\right)^{2}-f \Delta,
$$

where we used $d_{o}-\Delta / 2=\delta$.

Step 1 . We draw a circle with radius $\Delta / 2$. The diameter is the principal axis, and the object and image are the tangent lines. We draw a line parallel to the principal axis at the distance $\sqrt{f \Delta}$ - figure 3 (step 1).

Step 2. The parallel line has two intercepts with the circle: this is the location of the lens. Note that we have two solutions: the second solution corresponds to the case where the object and image are interchanged-figure 3 (step 2).

Step 3. We draw a circle with radius $f$ around the lens. The intercept with the principal axis yields the focal points $F_{1}$ and $F_{2}$-figure 3 (step 3 ).

Step 4. Given the location of the lens, the object and the two focal points $F_{1}$ and $F_{2}$, we draw rays 1,2, and 3 to confirm the location of the image-figure 3 (step 4).

Since the circle with a radius $\Delta / 2$ must intersect the line at the distance $\sqrt{f \Delta}$ from the principal axis, the geometric construction yields the inequality $\sqrt{f \Delta}>\Delta / 2$. We thus recover the condition between the object-image distance $\Delta$ and the focal length $f$.

The relationship between the focal length $f$ and the distance $\Delta$ is nontrivial, and is usually derived with calculus. We start from the lens equation. and write the image distance in terms of the object distance $d_{o}$ and the focal length $f: d_{i}=f d_{o} /\left(d_{o}-f\right)$. The distance between the object and image follows $\Delta=d_{o}+d_{i}$ :

$$
\Delta=\frac{d_{o}^{2}}{d_{o}-f}
$$

This equation gives the distance between the object and image as a function of the object distance $\Delta=\Delta\left(d_{o}\right)$. We find the maximum (or extrema) by taking the derivative with respect to $d_{o}$ :

$$
\frac{d \Delta}{d d_{o}}=\frac{2 d_{o}\left(d_{o}-f\right)-d_{o}^{2}}{\left(d_{o}-f\right)^{2}}=0 .
$$

The quadratic equation $2 d_{o}\left(d_{o}-f\right)-d_{o}^{2}=0$ has a trivial solution $d_{o}=0$ and a non-trivial solution

$$
d_{0}=2 f
$$

The image distance then follows $d_{i}=f \cdot 2 f /(2 f-f)=2 f$, so that $d_{i}=d_{o}$, and we recover the result $f_{\max }=\Delta / 4$. 

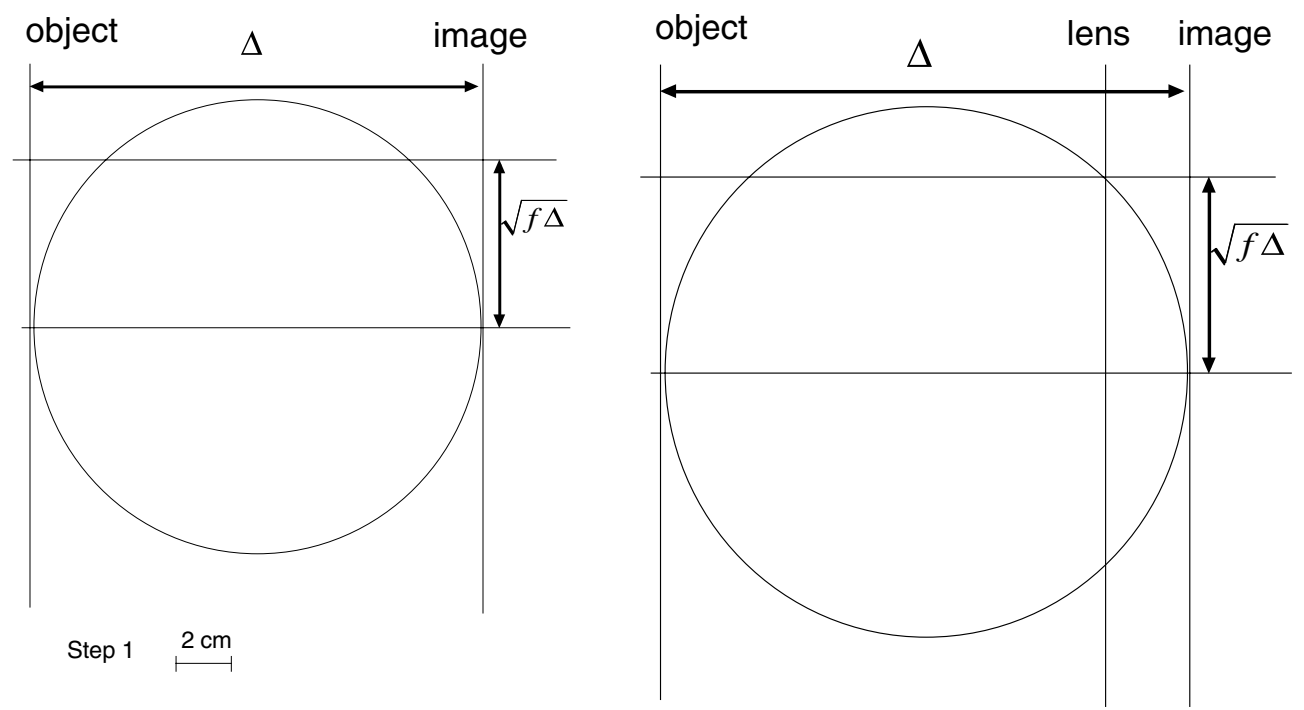

Step $2 \quad 2 \mathrm{~cm}$
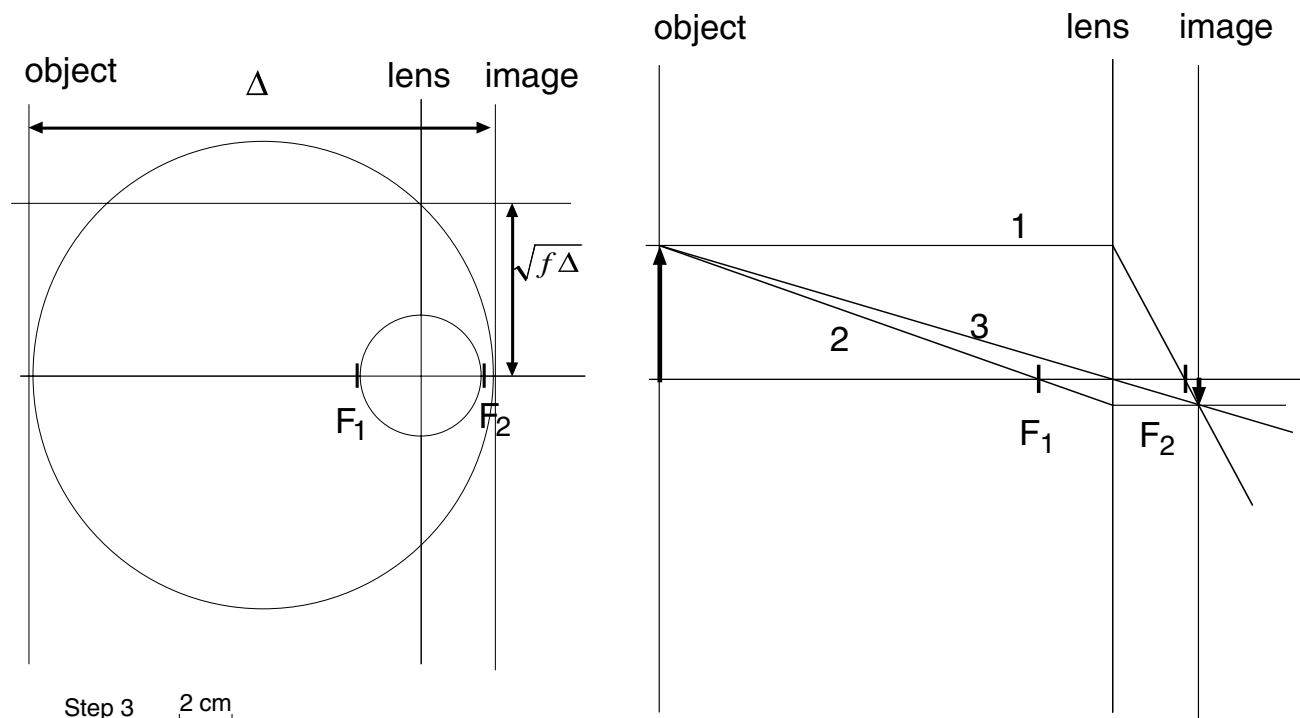

Step $3 \quad 2 \mathrm{~cm}$

Step $4 \stackrel{2 \mathrm{~cm}}{\longmapsto}$

Figure 3. The geometric construction for finding the location of the converging lens, if the distance between object and image $\Delta$, and the focal length $f$ are known. The construction applies to the case of a real image.

\section{Virtual image}

Similar to the above case, we draw a circle with a radius $\Delta / 2$. The principal axis goes through the centre of the circle. However, we cannot draw it (e.g., along the horizontal direction), since the lens is outside the circle. Thus, the first step of a geometric construction is the determination of the principal axis. 


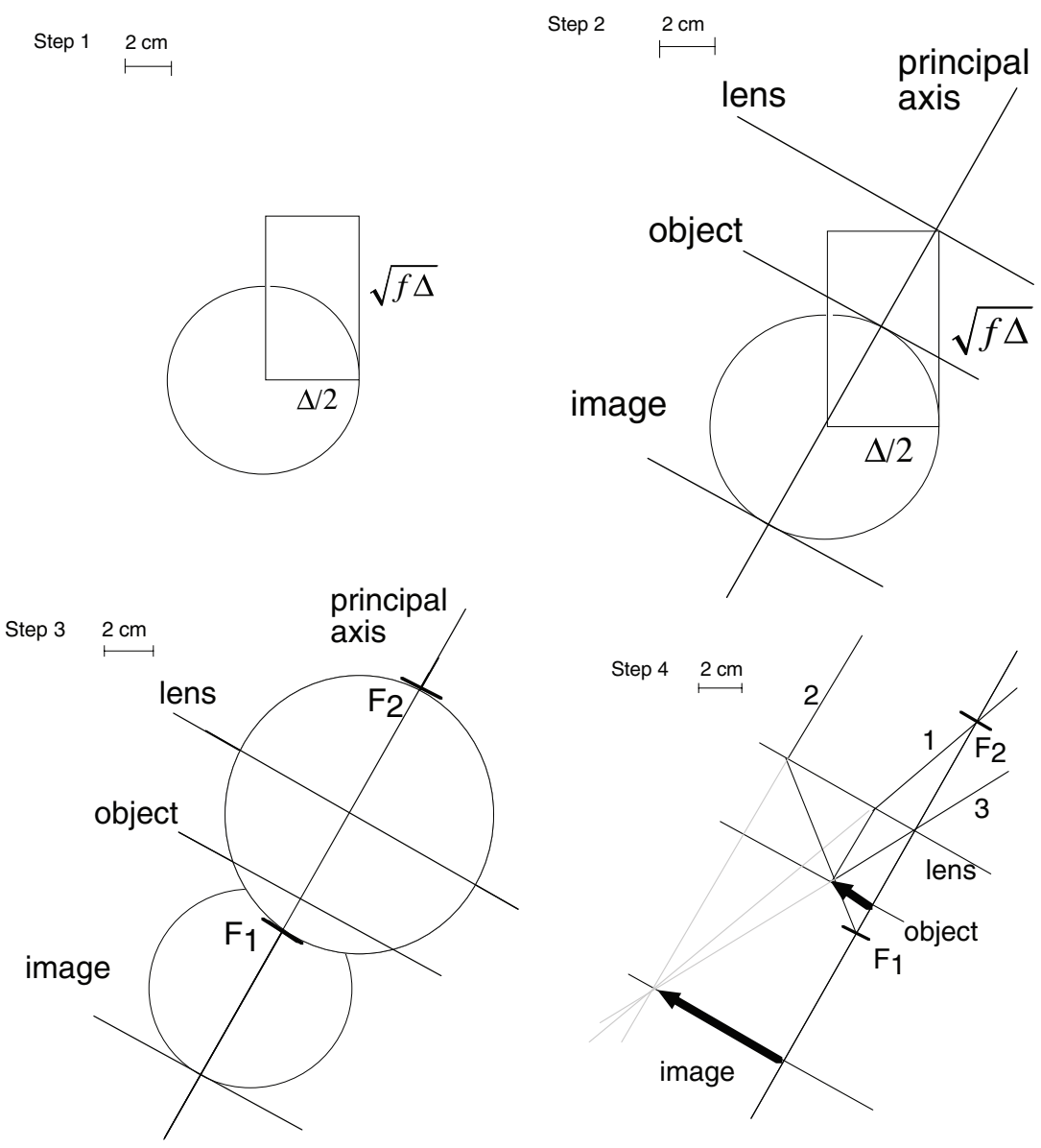

Figure 4. The geometric construction for finding the location of the converging lens, if the distance between object and image $\Delta$, and the focal length $f$ are known. The construction applies to the case of a virtual image.

Problem. Find the location of a lens with focal length $f$ if the distance $\Delta$ between the object and image are given. Numerical example: $\Delta=8.0 \mathrm{~cm}$ and $f=(49 / 8) \mathrm{cm}=6.125 \mathrm{~cm}$ so that $\sqrt{f \Delta}=7.0 \mathrm{~cm}$.

Solution. Since the image distance is negative $d_{i}<0$, the distance between the object and image follows $\Delta=-d_{o}-d_{i}$. Similar to the case for the real image, we draw a circle of radius $\Delta / 2$ so that the centre of the circle coincides with the midpoint between the object and image. We write $d_{i}=-\left(d_{o}+\Delta\right)$ so that $1 / d_{o}-1 /\left(d_{o}+\Delta\right)=1 / f$. It follows $f \Delta=d_{0}\left(d_{o}+\Delta\right)$ or by completing the square,

$$
\delta^{\prime 2}=\left(\frac{\Delta}{2}\right)^{2}+f \Delta,
$$

where $\delta^{\prime}=\Delta / 2+d_{o}$ is the distance between the lens and the centre of the circle.

Step 1 . We draw a rectangle with sides $\Delta / 2$ and $\sqrt{f \Delta}$. We draw a circle with radius $\Delta / 2$, which is centred at one of the corners of the rectangle-figure 4 (step 1). 
Step 2. We identify the diagonal line with the principal axis. The corner of the rectangle indicates the location of the lens. The intercepts of the circle with the principal axis yield the object and image planes-figure 4 (step 2).

Step 3. We draw a circle with radius $f$ around the lens. This gives us the focal points $F_{1}$ and $F_{2}$-figure 4 (step 3). Note that the object is inside the focal length of the lens.

Step 4 . We remove the two circles for clarity. Given the location of the lens, the object and the two focal points $F_{1}$ and $F_{2}$, we draw rays 1,2 and 3 to confirm the location of the image-figure 4 (step 4).

We see that any combination of object-image distance $\Delta$ and focal length $f$ is a possible solution. That is, there is no restriction for the focal length, as is the case for a real image. We observe furthermore that $d_{o}$ increases monotonically with $\Delta$. This implies that the objectimage distances increases, $\Delta \rightarrow \infty$, as the object moves towards the focal point and the object distance approaches the focal length from below for $d_{o} \rightarrow f^{-}$. We derive this result from the lens equation. For $d_{o}<f$, we write $d_{i}=-f d_{o} /\left(f-d_{o}\right)$. Thus, we have for the distance between object and image: $\Delta=f d_{0} /\left(f-d_{o}\right)-d_{o}$ so that we recover $\Delta \rightarrow \infty$ as $d_{o} \rightarrow f^{-}$.

\section{Discussion}

We discussed the geometric constructions for image formation using ruler and compass; the procedures can be generalized to the cases of a diverging lens and convex/concave mirrors. These constructions emphasize the geometric character of special rays 1,2 and 3, introduced in introductory texts.

We frequently use problems from section 2 (i.e. those that involve only a ruler) for assessment (exams). We usually choose numerical values so that the construction fits on a $8 \frac{1}{2} \times 11$ letter size paper and students do not have to rescale all length scales before drawing the appropriate lines. We do not find it necessary to distribute graph paper; students generally find an accurate construction by drawing parallel and perpendicular lines by sight. It generally requires a 'good' choice of the numerical values for $d_{o}, d_{i}, \Delta$ and $m$ so that angles between intersecting lines are not too small. This geometric problem solving forces students to first gain a qualitative understanding of the relationship between the object, image and lens before they start with the actual drawing. Students obtain results that are correct to within $10 \%$ of the correct value; this is, of course, not because of an inherent limitation of the geometric method, since it as precise as the algebraic method. Rather the approximate nature is due to the limitations of drawing parallel and perpendicular lines with limited accuracy.

Intuition is built when students are exposed to several different views of the same problem. In geometric optics, three different views are available: hands-on laboratory experience, algebraic methods involving the mirror/lens and magnification equations, and geometric methods described in this paper. We hope that this paper shows that geometric methods should play a greater role in introductory physics courses. The informal response from students has been overwhelmingly positive: they first find a geometric solution of a problem from image formation before reverting to the algebraic method using the lens/mirror equation.

\section{Acknowledgments}

We wish to thank two anonymous referees for their constructive criticism of an earlier version of the manuscript. 


\section{References}

[1] See Adamson P 2007 Al-Kindi (Great Medieval Thinkers Series) (New York: Oxford University Press)

[2] Walker J 2011 Fundamentals of Physics-Halliday/Resnick/Walker 8th edn (New York: Wiley)

[3] Cutnell J D and Johnson K W 2012 Physics 9th edn (New York: Wiley)

[4] Goldberg F M and McDermott L C 1987 Am. J. Phys. 55108

[5] Coxeter H S M and Greitzer S L 1967 Geometry Revisited (Washington, DC: Mathematical Association of America)

[6] Courant R and Robbins H 1996 What is Mathematics? ed I Stewart (New York: Oxford University Press)

Post-print standardized by MSL Academic Endeavors, the imprint of the Michael Schwartz Library at Cleveland State University, 2017. 